\title{
Fundamental solutions for the coupled extension-flexure laminate problem
}

\author{
S. Syngellakis \\ Wessex Institute, UK
}

\begin{abstract}
The subject matter of this paper relates to general laminates comprising orthotropic layers with arbitrarily oriented material axes. Fundamental solutions are derived for the thin laminated plate theory based on Kirchhoff hypothesis. The analysis relies on Fourier transforms whose inverses are obtained using contour complex variable integration of analytic functions. This process allows the derivation of explicit and compact forms for the fundamental solutions which are subsequently introduced into suitable reciprocity relations to obtain boundary integral equations for the general laminate coupled extension-flexure problem. Keywords: fundamental solutions, general laminate, Fourier transform, residue theorem.
\end{abstract}

\section{Introduction}

General analytical solutions for laminated plates, based on the classical lamination theory, are rather rare and restricted to rectangular plate domains. Provided that the fundamental solutions of the respective governing partial differential equations are available, numerical solutions accounting for the possible coupling between extensional and flexural deformation can be obtained by the boundary element method (BEM), which would also deal with plates of arbitrary shape.

Previous nonlinear BEM analyses of general laminates [1, 2] relied on the fundamental solutions of the two uncoupled, extensional and flexural problems. Compact expressions for these solutions and the other kernels arising from them are given in earlier articles on BEM applications to the symmetrically laminated plate problem [3]. The integral equations of the respective BEM formulations of the general laminate problem included irreducible domain integrals requiring 
additional domain modelling for the generation of a consistent system of algebraic equations.

For a genuine BEM solution, the fundamental solutions for the coupled problem are therefore essential. A first attempt at deriving such solutions was made by Becker [4] by representing the three displacement components in terms of complex potentials and their derivatives; the rather laborious process required the determination of four complex constants satisfying eight equilibrium and continuity conditions. No explicit forms of the fundamental solutions and their derivatives were obtained although plots of associated forces and moments were provided. More recently, Hwu [5] adopted a similar approach with the stress resultants being represented in terms of stress functions; this process led to fundamental solutions in explicit forms but still requiring expressions of considerable complexity for their description. A corresponding BEM formulation of the coupled extensional-flexural problem was developed by the same author [6].

In this paper, an alternative approach for the derivation of the fundamental solutions of the general laminate problem is adopted. The procedure is based on Fourier transforms and complex function integration. The problem is formulated in terms of the in-plane displacements and lateral deflection. In contrast to the solutions mentioned above, which applied unit moments about each co-ordinate direction, a unit moment about a single arbitrary in-plane direction was considered in the present analysis. Explicit and compact forms for the two inplane displacements, the deflection and its gradient relative to an arbitrary direction were obtained. These solutions are more consistent with classical formulations of plate stretching and bending problems; they were subsequently introduced into an appropriate reciprocity relation to yield boundary integral equations that can be solved by the standard BEM procedure.

\section{Theory}

The derivation of the classical lamination theory was based on Kirchhoff's thin plate assumptions. The theory comprises the constitutive equations

$$
\begin{gathered}
N_{\alpha \beta}=A_{\alpha \beta \gamma \delta} \varepsilon_{\gamma \delta}+B_{\alpha \beta \gamma \delta} \kappa_{\gamma \delta} \\
M_{\alpha \beta}=B_{\alpha \beta \gamma \delta} \varepsilon_{\gamma \delta}+D_{\alpha \beta \gamma \delta} \kappa_{\gamma \delta},
\end{gathered}
$$

the in-plane strain-displacement and curvature-deflection relations

$$
\begin{gathered}
\varepsilon_{\alpha \beta}=1 / 2\left(u_{\alpha, \beta}+u_{\beta, \alpha}\right) \\
\kappa_{\alpha \beta}=-w, \alpha \beta,
\end{gathered}
$$

and the equilibrium equations

$$
\begin{gathered}
N_{\alpha \beta, \beta}+f_{\alpha}=0 \\
M_{\alpha \beta, \alpha \beta}+q=0 .
\end{gathered}
$$


Tensor notation and an $x_{1}-x_{2}$ Cartesian frame of reference have been used so that the membrane forces are represented by $N_{\alpha \beta}$, the bending moments by $M_{\alpha \beta}$, the mid-plane strains by $\varepsilon_{\alpha \beta}$ and the curvatures by $\kappa_{\alpha \beta}$, the in-plane displacements by $u_{\alpha}$ and the transverse deflection by $w$, where all Greek indices range from 1 to 2 and the summation convention over repeated indices has been adopted. Moreover, a comma followed by a subscript represents differentiation with respect to the corresponding co-ordinate; for example, $u, \alpha$ means $\partial u / \partial x_{\alpha}$. In eqns (1) and (2), the material constants $A_{\alpha \beta \gamma \delta}$ are the extensional stiffness coefficients, $D_{\alpha \beta \gamma \delta}$ the flexural stiffness coefficients and $B_{\alpha \beta \gamma \delta}$ the bendingstretching coupling coefficients. In eqns (5) and (6), $f_{\alpha}$ and $q$ are the in-plane body forces and the lateral pressure, respectively.

Substitution of eqns (3) and (4) into eqns (1) and (2) allows the constitutive equations to be written in terms of in-plane displacements and deflection:

$$
\begin{gathered}
N_{\alpha \beta}=A_{\alpha \beta \gamma \delta u_{\gamma, \delta}-B_{\alpha \beta \gamma \delta} w, \gamma \delta} \\
M_{\alpha \beta}=B_{\alpha \beta \gamma \delta} u_{\gamma, \delta}-D_{\alpha \beta \gamma \delta} w, \gamma \delta
\end{gathered}
$$

Finally, substitution of eqns (7) and (8) into eqns (5) and (6) allows the equilibrium equations to be written in terms of in-plane displacements and deflection:

$$
\begin{aligned}
& A_{\alpha \beta \gamma \delta} u_{\gamma, \beta \delta}-B_{\alpha \beta \gamma \delta} w, \beta \gamma \delta \\
& -B_{\alpha \beta \gamma \delta} u_{\gamma, \alpha \beta \delta}+D_{\alpha \beta \gamma \delta}=0 \\
& w, \alpha \beta \gamma \delta
\end{aligned}
$$

The objective, in this paper, is the derivation of the fundamental solutions for the linear system of differential eqns (9) and (10).

\section{Fundamental solutions}

\subsection{In-plane extension}

The fundamental solution $u_{\lambda \gamma}^{*}, \tilde{w}_{\lambda}^{*}$ in this case satisfies

$$
\begin{gathered}
A_{\alpha \beta \gamma \delta} u_{\lambda \gamma, \beta \delta}^{*}-B_{\alpha \beta \gamma \delta} \tilde{w}_{\lambda, \beta \gamma \delta}^{*}+\delta_{\alpha \lambda} \delta(\mathbf{x}-\xi)=0 \\
-B_{\alpha \beta \gamma \delta} u_{\lambda \gamma, \alpha \beta \delta}^{*}+D_{\alpha \beta \gamma \delta} \tilde{w}_{\lambda,{ }_{\alpha \beta \gamma \delta}^{*}}^{*}=0
\end{gathered}
$$

where $\mathbf{x}\left(x_{1}, x_{2}\right)$ is the field point, $\xi\left(\xi_{1}, \xi_{2}\right)$ the source point in an infinite domain $\Omega_{\infty}$, as shown in fig. $1 ; \delta_{\alpha \beta}$ is the Kronecker delta and $\delta(\mathbf{x}-\xi)$ the Dirac delta function so that the product $\delta_{\alpha \lambda} \delta(\mathbf{x}-\xi)$ represents unit in-plane forces acting along $x_{\lambda}$ at $\xi\left(\xi_{1}, \xi_{2}\right)$. 


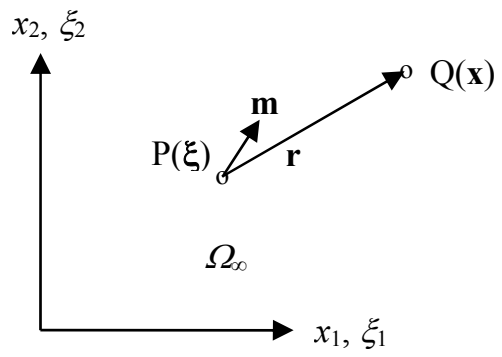

Figure 1: Infinite laminated plate under unit forces/moment at source point $\mathrm{P}(\xi)$.

In a two-dimensional space, the Fourier transforms of the dependent variables are given by [7]

$$
\begin{aligned}
& \hat{u}_{\lambda \gamma}=\frac{1}{2 \pi} \int_{\Omega_{\infty}} u_{\lambda \gamma}^{*} \mathrm{e}^{\mathrm{i} \zeta \cdot \mathrm{x}} \mathrm{d} \Omega \\
& \hat{w}_{\lambda}=\frac{1}{2 \pi} \int_{\Omega_{\infty}} \tilde{w}_{\lambda}^{*} \mathrm{e}^{\mathrm{i} \zeta \cdot \mathrm{x}} \mathrm{d} \Omega
\end{aligned}
$$

where $\zeta\left(\zeta_{1}, \zeta_{2}\right)$ represents the independent variable vector in the Fourier space. Taking the Fourier transforms of the left-hand sides of eqns (11) and (12) leads to the algebraic system of equations

$$
\begin{gathered}
-A \alpha \beta \gamma \delta \zeta_{\beta} \zeta_{\delta} \hat{u}_{\lambda \gamma}-\mathrm{i} B_{\alpha \beta \gamma \delta} \zeta_{\beta} \zeta_{\gamma} \zeta_{\delta} \hat{w}_{\lambda}+\frac{\delta_{\lambda \alpha}}{2 \pi} \mathrm{e}^{\mathrm{i} \zeta \cdot \xi}=0 \\
-\mathrm{i} B \alpha \beta \gamma \delta \zeta_{\alpha} \zeta_{\beta} \zeta_{\delta} \hat{u}_{\lambda \gamma}+D_{\alpha \beta \gamma \delta} \zeta_{\alpha} \zeta_{\beta} \zeta_{\gamma} \zeta_{\delta} \hat{w}_{\lambda}=0
\end{gathered}
$$

for the three transformed displacement functions associated with a unit force in the $x_{\lambda}$ direction. For more clarity, the system of eqns (13) and (14) is also written in matrix form:

$$
\left[\begin{array}{ccc}
A_{1 \alpha 1 \gamma} \zeta_{\alpha} \zeta_{\gamma} & A_{1 \beta 2 \delta} \zeta_{\beta} \zeta_{\delta} & \mathrm{i} B_{1 \eta \kappa} \zeta_{\kappa} \zeta_{\eta} \zeta_{\theta} \\
A_{2 \alpha 1 \gamma} \zeta_{\alpha} \zeta_{\gamma} & A_{2 \beta 2 \delta} \zeta_{\beta} \zeta_{\delta} & \mathrm{i} B_{2 \eta \kappa} \zeta_{\kappa} \zeta_{\eta} \zeta_{\theta} \\
-\mathrm{i} B_{\lambda \mu 1 v} \zeta_{\lambda} \zeta_{\mu} \zeta_{v} & -\mathrm{i} B_{\lambda \mu 2 \nu} \zeta_{\lambda} \zeta_{\mu} \zeta_{v} & D_{\kappa \lambda \mu \nu} \zeta_{\kappa} \zeta_{\lambda} \zeta_{\mu} \zeta_{v}
\end{array}\right]\left\{\begin{array}{c}
\hat{u}_{\lambda 1} \\
\hat{u}_{\lambda 2} \\
\hat{w}_{\lambda}
\end{array}\right\}=\left\{\begin{array}{c}
\delta_{\lambda 1} \\
\delta_{\lambda 2} \\
0
\end{array}\right\} \frac{\mathrm{e}^{\mathrm{i} \zeta \cdot \xi}}{2 \pi}
$$

so that its solution can be expressed in terms of determinants in the form

$$
\hat{u}_{\lambda 1}=\frac{1}{\Delta}\left|\begin{array}{ccc}
\delta_{\lambda 1} & A_{1 \beta 2 \delta} \zeta_{\beta} \zeta_{\delta} & \mathrm{i} B_{1 \eta \kappa} \zeta_{\kappa} \zeta_{\eta} \zeta_{\theta} \\
\delta_{\lambda 2} & A_{2 \beta 2 \delta} \zeta_{\beta} \zeta_{\delta} & \mathrm{i} B_{2 \eta \kappa} \zeta_{\kappa} \zeta_{\eta} \zeta_{\theta} \\
0 & -\mathrm{i} B_{\lambda \mu 2 v} \zeta_{\lambda} \zeta_{\mu} \zeta_{v} & D_{\kappa \lambda \mu \nu} \zeta_{\kappa} \zeta_{\lambda} \zeta_{\mu} \zeta_{v}
\end{array}\right| \begin{aligned}
& \mathrm{e}^{\mathrm{i} \zeta \cdot \xi} \\
& 2 \pi
\end{aligned}
$$




$$
\begin{gathered}
\hat{u}_{\lambda 2}=\frac{1}{\Delta}\left|\begin{array}{ccc}
A_{1 \alpha 1 \gamma} \zeta_{\alpha} \zeta_{\gamma} & \delta_{\lambda 1} & \mathrm{i} B_{1 \eta \kappa} \zeta_{\kappa} \zeta_{\eta} \zeta_{\theta} \\
A_{2 \alpha 1 \gamma} \zeta_{\alpha} \zeta_{\gamma} & \delta_{\lambda 2} & \mathrm{i} B_{2 \eta \kappa} \zeta_{\kappa} \zeta_{\eta} \zeta_{\theta} \\
\mathrm{i} B_{\lambda \mu 1 \nu} \zeta_{\lambda} \zeta_{\mu} \zeta_{v} & 0 & D_{\kappa \lambda \mu \nu} \zeta_{\kappa} \zeta_{\lambda} \zeta_{\mu} \zeta_{v}
\end{array}\right| \\
\hat{w}_{\lambda}=\frac{1}{\Delta}\left|\begin{array}{ccc}
A_{1 \alpha 1 \gamma} \zeta_{\alpha} \zeta_{\gamma} & A_{1 \beta 2 \delta} \zeta_{\beta} \zeta_{\delta} & \delta_{\lambda 1} \\
A_{2 \alpha 1 \gamma} \zeta_{\alpha} \zeta_{\gamma} & A_{2 \beta 2 \delta} \zeta_{\beta} \zeta_{\delta} & \delta_{\lambda 2} \\
-\mathrm{i} B_{\lambda \mu 1 v} \zeta_{\lambda} \zeta_{\mu} \zeta_{v} & -\mathrm{i} B_{\lambda \mu 2 \nu} \zeta_{\lambda} \zeta_{\mu} \zeta_{v} & 0
\end{array}\right| \frac{\mathrm{e}^{\mathrm{i} \zeta \cdot \xi}}{2 \pi}
\end{gathered}
$$

where $\Delta$ is the determinant of the coefficient matrix on the left hand side of eqn (15). Expansion of this determinant gives

$$
\begin{aligned}
\Delta\left(\zeta_{1}, \zeta_{2}\right) & =\left[\left(A_{1 \alpha 1 \gamma} A_{2 \beta 2 \delta-} A_{2 \alpha 1 \gamma} A_{1 \beta 2 \delta}\right) D_{\kappa \lambda \mu v}-\left(A_{1 \alpha 1 \gamma} B_{2 \beta \kappa \delta-} A_{2 \alpha 1 \gamma} B_{1 \beta \kappa \delta}\right) B \lambda \mu 2 v\right. \\
& \left.+\left(A_{1 \alpha 2 \gamma} B_{2 \beta \kappa \delta} A_{2 \alpha 2 \gamma} B_{1 \beta \kappa \delta}\right) B_{\lambda \mu 1 v}\right] \zeta_{\alpha} \zeta_{\beta} \zeta_{\gamma} \zeta_{\delta} \zeta_{\kappa} \zeta_{\lambda} \zeta_{\mu} \zeta_{v}
\end{aligned}
$$

Thus $\Delta\left(\zeta_{1}, \zeta_{2}\right)$ is an $8^{\text {th }}$ order polynomial in either $\zeta_{1}$ or $\zeta_{2}$ whose coefficients are expressed in terms of the material constants. Expansion of the determinants on the right-hand sides of eqns (16)-(18) results in similar polynomial expressions $g_{\lambda \lambda}\left(\zeta_{1}, \zeta_{2}\right)$ and $h_{\lambda}\left(\zeta_{1}, \zeta_{2}\right)$, of $6^{\text {th }}$ and $5^{\text {th }}$ order, respectively, so that

$$
\begin{gathered}
\hat{u}_{\lambda \gamma}=\frac{g_{\lambda \gamma}\left(\zeta_{1}, \zeta_{2}\right)}{\Delta\left(\zeta_{1}, \zeta_{2}\right)} \frac{\mathrm{e}^{\mathrm{i} \zeta \cdot \xi}}{2 \pi} \\
\hat{w}_{\lambda}=\frac{\mathrm{i} h_{\lambda}\left(\zeta_{1}, \zeta_{2}\right)}{\Delta\left(\zeta_{1}, \zeta_{2}\right)} \frac{\mathrm{e}^{\mathrm{i} \zeta \cdot \xi}}{2 \pi}
\end{gathered}
$$

The fundamental solutions can now be obtained as the inverses of their Fourier transforms given by eqns (20) and (21):

$$
\begin{aligned}
u_{\lambda \gamma}^{*}(\mathbf{x}, \xi) & =\frac{1}{2 \pi} \int_{\Omega_{\infty}} \hat{u}_{\lambda \gamma} \mathrm{e}^{-\mathrm{i} \zeta \cdot \mathrm{x}} \mathrm{d} \Omega=\frac{1}{4 \pi^{2}} \int_{\Omega_{\infty}} \frac{g_{\lambda \gamma}\left(\zeta_{1}, \zeta_{2}\right)}{\Delta\left(\zeta_{1}, \zeta_{2}\right)} \mathrm{e}^{-\mathrm{i} \zeta \cdot(\mathbf{x}-\xi)} \mathrm{d} \Omega(\zeta) \\
\tilde{w}_{\lambda}^{*}(\mathbf{x}, \boldsymbol{\xi}) & =\frac{1}{2 \pi} \int_{\Omega_{\infty}} \hat{w}_{\lambda} \mathrm{e}^{-\mathrm{i} \zeta \cdot \mathbf{x}} \mathrm{d} \Omega=\frac{1}{4 \pi^{2}} \int_{\Omega_{\infty}} \frac{\mathrm{i} h_{\lambda}\left(\zeta_{1}, \zeta_{2}\right)}{\Delta\left(\zeta_{1}, \zeta_{2}\right)} \mathrm{e}^{-\mathrm{i} \zeta \cdot(\mathbf{x}-\xi)} \mathrm{d} \Omega(\zeta)
\end{aligned}
$$

The integrations with respect to $\zeta$ on the right-hand sides of eqns (22) and (23) are performed by separation of variables, that is,

$$
\begin{aligned}
& u_{\lambda \gamma}^{*}=\frac{1}{4 \pi^{2}} \int_{-\infty}^{\infty} \mathrm{e}^{-\mathrm{i} \zeta_{1} r_{1}} \mathrm{~d} \zeta_{1} \int_{-\infty}^{\infty} \frac{g_{\lambda \gamma}\left(\zeta_{1}, \zeta_{2}\right)}{\Delta\left(\zeta_{1}, \zeta_{2}\right)} \mathrm{e}^{-\mathrm{i} \zeta_{2} r_{2}} \mathrm{~d} \zeta_{2} \\
& \tilde{w}_{\lambda}^{*}=\frac{\mathrm{i}}{4 \pi^{2}} \int_{-\infty}^{\infty} \mathrm{e}^{-\mathrm{i} \zeta_{1} r_{1}} \mathrm{~d} \zeta_{1} \int_{-\infty}^{\infty} \frac{h_{\lambda}\left(\zeta_{1}, \zeta_{2}\right)}{\Delta\left(\zeta_{1}, \zeta_{2}\right)} \mathrm{e}^{-\mathrm{i} \zeta_{2} r_{2}} \mathrm{~d} \zeta_{2}
\end{aligned}
$$

where $r_{\alpha}=x_{\alpha}-\xi_{\alpha}$. Integration with respect to $\zeta_{2}$ is performed using complex variable calculus [8]. Considering first the solution for the in-plane displacements, a complex variable $z=\zeta_{2}+\mathrm{i} \zeta^{\prime}$ is introduced and a complex function $F(z)$ is defined as

$$
F(z)=\frac{g_{\lambda \gamma}\left(\zeta_{1}, z\right)}{\Delta\left(\zeta_{1}, z\right)} \mathrm{e}^{-\mathrm{i} r_{2} z}
$$


which is integrated around the contour shown in fig. 2 comprising the segment of the real axis from $-R$ to $R$ and the semicircle $C_{R}$ in the upper half of the complex plane.

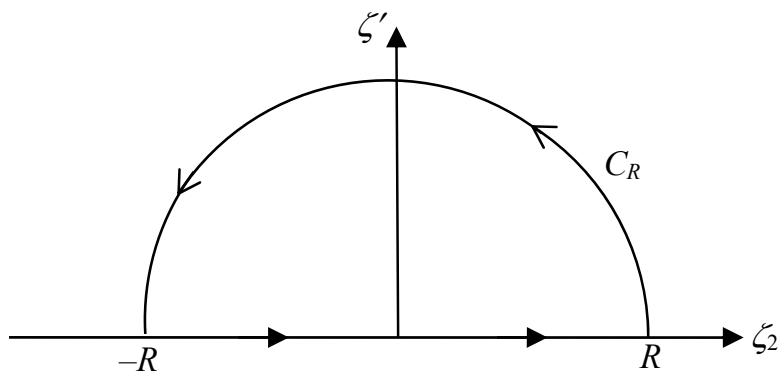

Figure 2: Contour for complex function integration.

Thus, for any value of $R$,

$$
\int_{-R}^{R} \frac{g_{\lambda \gamma}\left(\zeta_{1}, \zeta_{2}\right)}{\Delta\left(\zeta_{1}, \zeta_{2}\right)} \mathrm{e}^{-\mathrm{i} \zeta_{2} r_{2}} \mathrm{~d} \zeta_{2}+\int_{C_{R}} F(z) \mathrm{d} z=2 \pi \mathrm{i} \sum_{k} \operatorname{Res}\left(a_{k}\right)
$$

where $a_{k}$ represent the poles of $F(z)$ within the contour. It has been shown [8] that the contour integral along the semi-circle on the left-hand side tends to zero as $R$ goes to infinity provided that $r_{2}<0$. As $R$ increases, the semicircle expands and eventually encompasses all the poles of $F(z)$. To determine the residues associated with these poles, it is noted that the $8^{\text {th }}$ order polynomial $\Delta\left(\zeta_{1}, z\right)$ has four pairs of distinct complex conjugate roots [9] which can be easily expressed in terms of $\zeta_{1}$ by introducing the parameter $\rho$ such that $z=\zeta_{2}=\rho \zeta_{1}$; this transforms eqn (19) into

$$
\Delta\left(\zeta_{1}, z\right)=\zeta_{1}^{8} \Delta(\rho)
$$

The roots of $\Delta(\rho)$ are represented by

$$
\rho_{k}=\rho_{k}^{\prime}+\mathrm{i} \rho_{k}^{\prime \prime}, \bar{\rho}_{k}=\rho_{k}^{\prime}-\mathrm{i} \rho_{k}^{\prime \prime}, \rho_{k}^{\prime \prime}>0 ; k=1,2,3,4
$$

and the denominator in eqn (24) can thus be written

$$
\Delta\left(\zeta_{1}, \zeta_{2}\right)=\Delta_{8} \prod_{i=1}^{4}\left(\zeta_{2}-\rho_{i} \zeta_{1}\right)\left(\zeta_{2}-\bar{\rho}_{i} \zeta_{1}\right)
$$

where $\Delta_{8}$ is the coefficient of $\rho^{8}$ which can be expressed explicitly in terms of material constants. Since $\zeta_{1}$ varies from $-\infty$ to $\infty$ in the subsequent integration, it is, at this stage, necessary to distinguish between $\zeta_{1}$ being positive or negative.

(a) $\zeta_{1}>0$

In this case, $F(z)$ has four poles $\rho_{k} \zeta_{1}$ of order 1 (simple poles) in the upper half-plane and the respective residues are

$$
\operatorname{Res}\left(\rho_{k} \zeta_{1}\right)=\left[\left(z-\rho_{k} \zeta_{1}\right) F(z)\right]_{z=\rho_{k} \zeta_{1}}=\frac{g_{\lambda \gamma}\left(\rho_{k}\right) e^{-\mathrm{i} r_{2} \rho_{k} \zeta_{1}}}{2 \mathrm{i} \Delta_{8} \beta_{k} \zeta_{1}}
$$


where

$$
\beta_{k}=\rho_{k}^{\prime \prime} \prod_{i=1, i \neq k}^{4}\left(\rho_{k}-\rho_{i}\right)\left(\rho_{k}-\bar{\rho}_{i}\right)
$$

Thus

$$
\int_{-\infty}^{\infty} \frac{g_{\lambda \gamma}\left(\zeta_{1}, \zeta_{2}\right)}{\Delta\left(\zeta_{1}, \zeta_{2}\right)} \mathrm{e}^{-\mathrm{i} \zeta_{2} r_{2}} \mathrm{~d} \zeta_{2}=\frac{\pi}{\Delta_{8}} \sum_{k=1}^{4} \frac{g_{\lambda \gamma}\left(\rho_{k}\right) \mathrm{e}^{-\mathrm{i} r_{2} \rho_{k} \zeta_{1}}}{\beta_{k} \zeta_{1}}
$$

(b) $\zeta_{1}<0$

In this case, the complex conjugates of $\rho_{k}$, multiplied by $\zeta_{1}$, are the four simple poles of $F(z)$ in the upper half-plane; hence,

$$
\operatorname{Res}\left(\bar{\rho}_{k} \zeta_{1}\right)=\left[\left(z-\bar{\rho}_{k} \zeta_{1}\right) F(z)\right]_{z=\bar{\rho}_{k} \zeta_{1}}=\frac{g_{\lambda \gamma}\left(\bar{z}_{k}\right) e^{-\mathrm{i} \zeta_{1} r_{2} \bar{\rho}_{k}}}{-2 \mathrm{i} \Delta_{8} \bar{\beta}_{k} \zeta_{1}}
$$

and

$$
\int_{-\infty}^{\infty} \frac{g_{\lambda \gamma}\left(\zeta_{1}, \zeta_{2}\right)}{\Delta\left(\zeta_{1}, \zeta_{2}\right)} \mathrm{e}^{-\mathrm{i} \zeta_{2} r_{2}} \mathrm{~d} \zeta_{2}=-\frac{\pi}{\Delta_{8}} \sum_{k=1}^{4} \frac{g_{\lambda \gamma}\left(\bar{\rho}_{k}\right) \mathrm{e}^{-\mathrm{i} \zeta_{1} r_{2} \bar{\rho}_{k}}}{\bar{\beta}_{k} \zeta_{1}}
$$

It is thus possible to obtain the inverse transform given by eqn (22) over the whole range of $\zeta_{1}$ :

$$
u_{\lambda \gamma}^{*}=\frac{1}{4 \pi \Delta_{8}} \sum_{k=1}^{4} \frac{g_{\lambda \gamma}\left(\rho_{k}\right)}{\beta_{k}} \int_{0}^{\infty} \frac{\mathrm{e}^{-\mathrm{i} \zeta_{1} v_{k}}}{\zeta_{1}} \mathrm{~d} \zeta_{1}-\frac{1}{4 \pi \Delta_{8}} \sum_{k=1}^{4} \frac{g_{\lambda \gamma}\left(\bar{\rho}_{k}\right)}{\bar{\beta}_{k}} \int_{-\infty}^{0} \frac{\mathrm{e}^{-\mathrm{i} \zeta_{1} \bar{v}_{k}}}{\zeta_{1}} \mathrm{~d} \zeta_{1}
$$

where $v_{k}=r_{1}+\rho_{k} r_{2}$. By substituting $\zeta_{1}=s$ in the first integral of the right hand side of eqn (25) and $\zeta_{1}=-S$ in the second, it is possible to show that the second term is simply minus the complex conjugate of the first. This leads to the real expression

$$
u_{\lambda \gamma}^{*}=\frac{1}{2 \pi \Delta_{8}} \operatorname{Re}\left[\sum_{k=1}^{4} \frac{g_{\lambda \gamma}\left(\rho_{k}\right)}{\beta_{k}} \int_{0}^{\infty} \frac{\mathrm{e}^{-i s v_{k}}}{s} \mathrm{~d} s\right]
$$

Since $r_{2}<0, \operatorname{Re}\left(-\mathrm{i} v_{k}\right)<0$; thus $\exp \left(-\mathrm{i} v_{k} s\right)$ is bounded. Under this condition, the Cauchy principal value of this divergent integral can be obtained by making use of a property of the Laplace transforms [8], which leads to

$$
\mathrm{P} \int_{0}^{\infty} \frac{\mathrm{e}^{-i s v_{k}}}{s} \mathrm{~d} s=-\ln v_{k}
$$

Thus, finally, the fundamental solutions for the in-plane displacements is provided in the form of the real functions

$$
u_{\lambda \gamma}^{*}=-\frac{1}{2 \pi \Delta_{8}} \operatorname{Re}\left[\sum_{k=1}^{4} \frac{g_{\lambda \gamma}\left(\rho_{k}\right)}{\beta_{k}} \ln v_{k}\right]
$$

If $r_{2}>0$, for the contour integral over $C_{R}$ to tend to zero as $R$ goes to infinity, the semicircle should lie in the lower half-plane. The process described above is repeated leading to the same final result.

The inverse Fourier transform of the deflection given by eqn (23) is also integrated by separation of variables. Integration with respect to $\zeta_{2}$ is performed by the same process as that applied for the in-plane displacements with $g_{\lambda \gamma}$ 
replaced by $h_{\lambda}$. Since the latter is a $5^{\text {th }}$ order polynomial, the square of $\zeta_{1}$ appears in the denominator of the residues and the fundamental solution for the deflection becomes

$$
\tilde{w}_{\lambda}^{*}=\frac{\mathrm{i}}{4 \pi \Delta_{8}} \sum_{k=1}^{4} \frac{h_{\lambda}\left(\rho_{k}\right)}{\beta_{k}} \int_{0}^{\infty} \frac{\mathrm{e}^{-\mathrm{i} \zeta_{1} \nu_{k}}}{\zeta_{1}^{2}} \mathrm{~d} \zeta_{1}-\frac{\mathrm{i}}{4 \pi \Delta_{8}} \sum_{k=1}^{4} \frac{h_{\lambda}\left(\bar{\rho}_{k}\right)}{\beta_{k}} \int_{-\infty}^{0} \frac{\mathrm{e}^{-\mathrm{i} \zeta_{1} \bar{v}_{k}}}{\zeta_{1}^{2}} \mathrm{~d} \zeta_{1}
$$

Making again the substitutions $\zeta_{1}=s$ and $\zeta_{1}=-s$ in the first and second integral of the right hand side of the latter equation results in

$$
\tilde{w}_{\lambda}^{*}=\frac{1}{2 \pi \Delta_{8}} \operatorname{Re}\left[\sum_{k=1}^{4} \frac{\mathrm{i} h_{\lambda}\left(\rho_{k}\right)}{\beta_{k}} \int_{0}^{\infty} \frac{\mathrm{e}^{-\mathrm{i} s v_{k}}}{s^{2}} \mathrm{~d} s\right]
$$

Integration by parts and the application of the previously obtained result given by eqn (26), leads to

$$
\mathrm{P} \int_{0}^{\infty} \frac{\mathrm{e}^{-\mathrm{i} s v_{k}}}{s^{2}} \mathrm{~d} s=-\mathrm{i} v_{k} \ln v_{k}
$$

Hence the fundamental solution for the deflection due to in-plane forces is

$$
\tilde{w}_{\lambda}^{*}=\frac{1}{2 \pi \Delta_{8}} \operatorname{Re}\left[\sum_{k=1}^{4} \frac{h_{\lambda}\left(\rho_{k}\right) v_{k} \ln v_{k}}{\beta_{k}}\right]
$$

\subsection{Flexure}

The fundamental solution for plate bending satisfies

$$
\begin{gathered}
A_{\alpha \beta \gamma \delta} \tilde{u}_{\lambda \gamma, \beta \delta}^{*}-B_{\alpha \beta \gamma \delta} w_{\lambda, \beta \gamma \delta}^{*}=0 \\
-B_{\alpha \beta \gamma \delta} \tilde{u}_{\lambda \gamma, \alpha \beta \delta}^{*}+D_{\alpha \beta \gamma \delta} w_{\lambda}^{*},_{\alpha \beta \gamma \delta}=\delta_{\lambda}(\mathbf{x}-\xi)
\end{gathered}
$$

where

$$
\delta_{1}(\mathbf{x}-\xi)=\delta(\mathbf{x}-\xi) \text { and } \delta_{2}(\mathbf{x}-\xi)=\frac{\partial \delta(\mathbf{x}-\xi)}{\partial m(\xi)}
$$

The two infinite plate deflections and associated in-plane displacements at a field point $\mathrm{Q}(\mathbf{x})$ governed by eqns (30) and (31) are interpreted as [10]:

$\tilde{u}_{1 \gamma}^{*}, w_{1}^{*}$ are due to a transverse unit point force at the source point $\mathrm{P}(\xi)$,

$\tilde{u}_{2 \gamma}^{*}, w_{2}^{*}$ are due to a unit moment at $\mathrm{P}$ about the direction normal to unit vector $\mathbf{m}$ as schematically shown in fig. 1 .

Taking the Fourier transforms of both sides of eqns (30) and (31) gives

$$
\begin{gathered}
-A_{\alpha \beta \gamma \delta} \zeta_{\beta} \zeta_{\delta} \hat{u}_{\lambda \gamma}-\mathrm{i} B_{\alpha \beta \gamma \delta} \zeta_{\beta} \zeta_{\gamma} \zeta_{\delta} \hat{w}_{\lambda}=0 \\
-\mathrm{i} B_{\alpha \beta \gamma \delta} \zeta_{\alpha} \zeta_{\beta} \zeta_{\delta} \hat{u}_{\lambda \gamma}+D_{\alpha \beta \gamma \delta} \zeta_{\alpha} \zeta_{\beta} \zeta_{\gamma} \zeta_{\delta} \hat{w}_{\lambda}=\frac{b_{\lambda}}{2 \pi} \mathrm{e}^{\mathrm{i} \zeta \cdot \xi}
\end{gathered}
$$

where $b_{1}=1$ and $b_{2}=-\mathrm{i} \zeta_{k} m_{k}$. The solution of the system of eqns (32) and (33) gives the expressions 


$$
\begin{gathered}
\hat{u}_{\lambda \gamma}=\frac{\mathrm{i}}{2 \pi} \frac{\varphi_{\gamma}\left(\zeta_{1}, \zeta_{2}\right) b_{\lambda} \mathrm{e}^{\mathrm{i} \zeta \cdot \xi}}{\Delta\left(\zeta_{1}, \zeta_{2}\right)} \\
\hat{w}_{\lambda}=\frac{1}{2 \pi} \frac{\psi\left(\zeta_{1}, \zeta_{2}\right) b_{\lambda} \mathrm{e}^{\mathrm{i} \zeta \cdot \xi}}{\Delta\left(\zeta_{1}, \zeta_{2}\right)}
\end{gathered}
$$

where $\varphi_{\gamma}$ and $\psi$ are, respectively, $5^{\text {th }}$ and $4^{\text {th }}$ order polynomials in either $\zeta_{1}$ or $\zeta_{2}$ while $\Delta\left(\zeta_{1}, \zeta_{2}\right)$ is still given by eqn (19). The process of finding the inverse transforms of eqns (34) and (35) follows the same steps as in the case of extension. Thus, separation of variables gives

$$
\begin{gathered}
\tilde{u}_{\lambda \gamma}^{*}=\frac{\mathrm{i}}{4 \pi^{2}} \int_{-\infty}^{\infty} \mathrm{e}^{-\mathrm{i} \zeta_{1} r_{1}} \mathrm{~d} \zeta_{1} \int_{-\infty}^{\infty} \frac{\varphi_{\gamma}\left(\zeta_{1}, \zeta_{2}\right) b_{\lambda}\left(\zeta_{1}, \zeta_{2}\right)}{\Delta\left(\zeta_{1}, \zeta_{2}\right)} \mathrm{e}^{-\mathrm{i} \zeta_{2} r_{2}} \mathrm{~d} \zeta_{2} \\
w_{\lambda}^{*}=\frac{1}{4 \pi^{2}} \int_{-\infty}^{\infty} \mathrm{e}^{-\mathrm{i} \zeta_{1} r_{1}} \mathrm{~d} \zeta_{1} \int_{-\infty}^{\infty} \frac{\psi\left(\zeta_{1}, \zeta_{2}\right) b_{\lambda}\left(\zeta_{1}, \zeta_{2}\right)}{\Delta\left(\zeta_{1}, \zeta_{2}\right)} \mathrm{e}^{-\mathrm{i} \zeta_{2} r_{2}} \mathrm{~d} \zeta_{2}
\end{gathered}
$$

Contour integration with respect to $\zeta_{2}$ results in the following expressions

$$
\begin{array}{r}
\tilde{u}_{\lambda \gamma}^{*}=\frac{\mathrm{i}}{4 \pi \Delta_{8}} \sum_{k=1}^{4} \frac{\varphi_{\gamma}\left(\rho_{k}\right)}{\beta_{k}} \int_{0}^{\infty} \frac{b_{\lambda}\left(\zeta_{1}, \rho_{k} \zeta_{1}\right) \mathrm{e}^{-\mathrm{i} \zeta_{1} v_{k}}}{\zeta_{1}^{2}} \mathrm{~d} \zeta_{1} \\
\quad-\frac{\mathrm{i}}{4 \pi \Delta_{8}} \sum_{k=1}^{4} \frac{\varphi_{\gamma}\left(\bar{\rho}_{k}\right)}{\bar{\beta}_{k}} \int_{-\infty}^{0} \frac{b_{\lambda}\left(\zeta_{1}, \bar{\rho}_{k} \zeta_{1}\right) \mathrm{e}^{-\mathrm{i} \zeta_{1} \bar{v}_{k}}}{\zeta_{1}^{2}} \mathrm{~d} \zeta_{1} \\
w_{\lambda}^{*}=\frac{1}{4 \pi \Delta_{8}} \sum_{k=1}^{4} \frac{\psi\left(\rho_{k}\right)}{\beta_{k}} \int_{0}^{\infty} \frac{b_{\lambda}\left(\zeta_{1}, \rho_{k} \zeta_{1}\right) \mathrm{e}^{-\mathrm{i} \zeta_{1} v_{k}}}{\zeta_{1}^{3}} \mathrm{~d} \zeta_{1} \\
-\frac{1}{4 \pi \Delta_{8}} \sum_{k=1}^{4} \frac{\psi\left(\bar{\rho}_{k}\right)}{\bar{\beta}_{k}} \int_{-\infty}^{0} \frac{b_{\lambda}\left(\zeta_{1}, \bar{\rho}_{k} \zeta_{1}\right) \mathrm{e}^{-\mathrm{i} \zeta_{1} \bar{v}_{k}}}{\zeta_{1}^{3}} \mathrm{~d} \zeta_{1}
\end{array}
$$

For $\lambda=1$ and $b_{1}=1$, eqns (36) and (37) can be reduced to

$$
\begin{gathered}
\tilde{u}_{1 \gamma}^{*}=\frac{1}{4 \pi \Delta_{8}} \operatorname{Re}\left[\mathrm{i} \sum_{k=1}^{4} \frac{\varphi_{\gamma}\left(\rho_{k}\right)}{\beta_{k}} \int_{0}^{\infty} \frac{\mathrm{e}^{-\mathrm{is} v_{k}}}{s^{2}} \mathrm{~d} s\right] \\
w_{1}^{*}=\frac{1}{2 \pi \Delta_{8}} \operatorname{Re}\left[\sum_{k=1}^{4} \frac{\psi\left(\rho_{k}\right)}{\beta_{k}} \int_{0}^{\infty} \frac{\mathrm{e}^{-i s v_{k}}}{s^{3}} \mathrm{~d} s\right]
\end{gathered}
$$

Successive integration by parts and the use of relation (26) gives

$$
\mathrm{P} \int_{0}^{\infty} \frac{\mathrm{e}^{-\mathrm{i} s v_{k}}}{s^{3}} \mathrm{~d} s=\frac{v_{k}^{2}}{2} \ln v_{k}
$$

Hence, use of eqns (28) and (38) leads to the final form of the fundamental solutions for the displacements due to unit lateral force

$$
\tilde{u}_{1 \gamma}^{*}=\frac{1}{2 \pi \Delta_{8}} \operatorname{Re}\left[\sum_{k=1}^{4} \frac{\varphi_{\gamma}\left(\rho_{k}\right)}{\beta_{k}} v_{k} \ln v_{k}\right]
$$




$$
w_{1}^{*}=\frac{1}{4 \pi \Delta_{8}} \operatorname{Re}\left[\sum_{k=1}^{4} \frac{\psi\left(\rho_{k}\right)}{\beta_{k}} v_{k}^{2} \ln v_{k}\right]
$$

For $\lambda=2$ and $b_{2}=-\mathrm{i}\left(m_{1}+\rho_{k} m_{2}\right) \zeta_{1}$, eqns (36) and (37) are similarly transformed to

$$
\begin{aligned}
& \tilde{u}_{2 \gamma}^{*}=\frac{1}{2 \pi \Delta_{8}} \operatorname{Re}\left[\sum_{k=1}^{4} \frac{\varphi_{\gamma}\left(\rho_{k}\right)}{\beta_{k}}\left(m_{1}+m_{2} \rho_{k}\right) \int_{0}^{\infty} \frac{\mathrm{e}^{-\mathrm{i} v_{k} s}}{s} \mathrm{~d} s\right] \\
& w_{2}^{*}=-\frac{1}{2 \pi \Delta_{8}} \operatorname{Re}\left[\sum_{k=1}^{4} \frac{\mathrm{i} \psi\left(\rho_{k}\right)}{\beta_{k}}\left(m_{1}+m_{2} \rho_{k}\right) \int_{0}^{\infty} \frac{\mathrm{e}^{-\mathrm{i} v_{k} s}}{s^{2}} \mathrm{~d} s\right]
\end{aligned}
$$

Taking into account eqns (26) and (28) finally provides also the fundamental solutions for the displacements due to a unit moment about $\mathbf{m}$ :

$$
\begin{aligned}
& \tilde{u}_{2 \gamma}^{*}=-\frac{1}{2 \pi \Delta_{8}} \operatorname{Re}\left[\sum_{k=1}^{4} \frac{\varphi_{\gamma}\left(\rho_{k}\right)}{\beta_{k}}\left(m_{1}+m_{2} \rho_{k}\right) \ln v_{k}\right] \\
& w_{2}^{*}=-\frac{1}{2 \pi \Delta_{8}} \operatorname{Re}\left[\sum_{k=1}^{4} \frac{\psi\left(\rho_{k}\right)}{\beta_{k}}\left(m_{1}+m_{2} \rho_{k}\right) v_{k} \ln v_{k}\right]
\end{aligned}
$$

It can be noted that eqns (43) and (44) are, respectively, the singular parts of the derivatives of eqns (39) and (40) with respect to $\mathbf{m}$.

\section{Boundary integral equations}

BEM can now be applied to the analysis of a finite plate, whose domain $\Omega$ is bounded by the contour $\Gamma$ as shown in fig. 3. Apart for the in-plane body forces and the lateral pressure, the plate may also be subjected to various edge loads

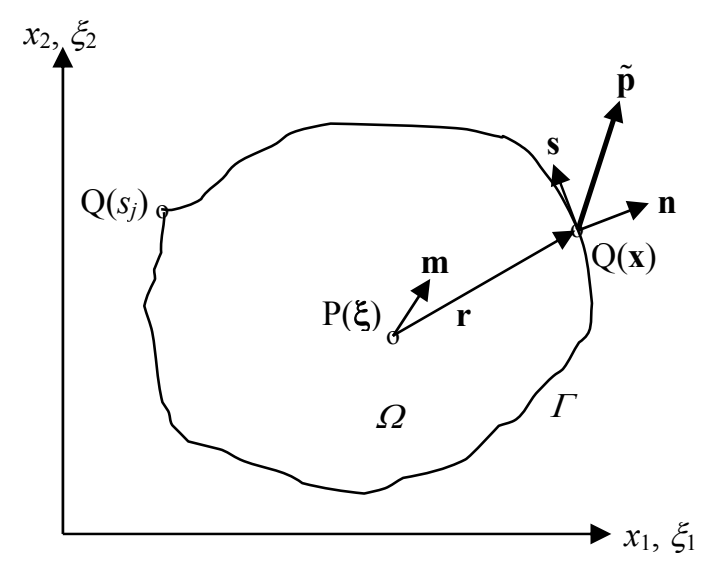

Figure 3: Laminate plate of arbitrary shape under edge traction $\tilde{\mathbf{p}}$. 
such as traction $\tilde{\mathbf{p}}$. A BEM solution process can be developed by formulating first the reciprocity relations

$$
\begin{aligned}
& \int_{\Omega}\left(N_{\alpha \beta} u_{\lambda \alpha, \beta}^{*}+M_{\alpha \beta} \tilde{w}_{\lambda, \alpha \beta}^{*}\right) \mathrm{d} \Omega=\int_{\Omega}\left[N_{\alpha \beta}\left(u_{\lambda \alpha}^{*}, \tilde{w}_{\lambda}^{*}\right) u_{\alpha, \beta}+M_{\alpha \beta}\left(u_{\lambda \alpha}^{*}, \tilde{w}_{\lambda}^{*}\right) w,_{\alpha \beta}\right] \mathrm{d} \Omega \\
& \int_{\Omega}\left(N_{\alpha \beta} \tilde{u}_{\lambda \alpha}^{*},_{\beta}+M_{\alpha \beta} w_{\lambda,{ }_{\alpha \beta}}^{*}\right) \mathrm{d} \Omega=\int_{\Omega}\left[N_{\alpha \beta}\left(\tilde{u}_{\lambda \alpha}^{*}, w_{\lambda}^{*}\right) u_{\alpha,,_{\beta}}+M_{\alpha \beta}\left(\tilde{u}_{\lambda \alpha}^{*}, w_{\lambda}^{*}\right) w,_{\alpha \beta}\right] \mathrm{d} \Omega
\end{aligned}
$$

for the extensional and flexural problem, respectively. Taking into account the constitutive relations for both the actual, coupled as well as the fundamental states, eqns (45) and (46) are transformed to

$$
\begin{gathered}
k u_{\lambda}(\xi)=I_{u}\left(u_{\alpha}, u_{\lambda \alpha}^{*}\right)-I_{w}\left(w, \tilde{w}_{\lambda}^{*}\right)-J\left(w, \tilde{w}_{\lambda}^{*}\right)+\int_{\Omega}\left(f_{\alpha} u_{\lambda \alpha}^{*}-q \tilde{w}_{\lambda}^{*}\right) \mathrm{d} \Omega \\
-k w_{\lambda}(\xi)=I_{u}\left(u_{\alpha}, \tilde{u}_{\lambda \alpha}^{*}\right)-I_{w}\left(w, w_{\lambda}^{*}\right)-J\left(w, w_{\lambda}^{*}\right)+\int_{\Omega}\left(f_{\alpha} \tilde{u}_{\lambda \alpha}^{*}-q w_{\lambda}^{*}\right) \mathrm{d} \Omega
\end{gathered}
$$

where $k=1$ or $1 / 2$, depending on whether $\mathrm{P}(\xi)$ lies within $\Omega$ or it belongs to smooth portions of $\Gamma$, respectively, and

$$
w_{1}=w, w_{2}=\frac{\partial w}{\partial n}
$$

The right-hand sides of eqns (47) and (48) comprise the boundary integrals as well as a jump term which are defined by the equations

$$
\begin{gathered}
I_{u}\left(u_{\alpha}, u_{\alpha}^{\prime}\right)=\int_{\Gamma}\left[p_{\alpha}\left(u_{\beta}\right) u_{\alpha}^{\prime}-p_{\alpha}\left(u_{\beta}^{\prime}\right) u_{\alpha}\right] \mathrm{d} s \\
I_{w}\left(w, w^{\prime}\right)=\int_{\Gamma}\left[V_{n}(w) w^{\prime}-M_{n}(w) \frac{\partial w^{\prime}}{\partial n}+M_{n}\left(w^{\prime}\right) \frac{\partial w}{\partial n}-V_{n}\left(w^{\prime}\right) w\right] \mathrm{d} s \\
J\left(w, w^{\prime}\right)=\sum_{j=1}^{K}\left[C_{j}(w) w_{j}^{\prime}-C_{j}\left(w^{\prime}\right) w_{j}\right]
\end{gathered}
$$

where force $C_{j}$ represents the discontinuity jump of twisting moment $M_{n s}$ at corner $j$ and $K$ is the number of corner points along $\Gamma$. Implicit expressions for the various boundary variables in terms of displacement components have been given in previous articles on laminate stability analyses [1, 2]. The domain integrals on the right-hand sides of eqns (47) and (48) depend on the applied loading and can be easily calculated by either $2 \mathrm{D}$ quadrature over the domain or by converting them to boundary integrals through dual reciprocity.

\section{Conclusions}

It has been shown that the Fourier transform approach can be very effective in producing the fundamental solutions of the coupled plate extension-flexure problem in explicit and compact forms which could be easily evaluated and used in a BEM analysis of an arbitrarily loaded, finite laminated plate of general shape and anisotropy. The basis of a BEM formulation has been provided here but considerable work is still required. This includes the derivation of the additional kernels that appear in the boundary and domain integrals of eqns (47) and (48), 
dealing with the complexities of boundary modelling and the development of a numerical algorithm that could be implemented through a computer program.

An initial validation of the derived solutions can be carried out by comparing their predictions with those of earlier analytical processes and formulas. The ultimate test for these fundamental solutions would be in the effectiveness of the corresponding boundary element formulations to provide reliable answers to practical laminated plate problems. One important type of failure of thin plates in engineering applications is buckling. The use of the new fundamental solutions will simplify earlier BEM stability and more general nonlinear analyses of laminates by removing the necessity of certain domain integrals although those due to nonlinearity will still remain irreducible.

\section{References}

[1] Syngellakis, S., A boundary element approach to buckling of general laminates. Boundary Elements and other Mesh Reduction Methods XXXIV, eds. C.A. Brebbia \& D. Poljak, WIT Press: Southampton, pp. 145-155, 2012.

[2] Syngellakis, S., Stability analysis for laminates with general anisotropy using boundary elements. Boundary Elements and other Mesh Reduction Methods $X X X V$, eds. C.A. Brebbia \& A.H.-D. Cheng, WIT Press: Southampton, pp. 133-144, 2013.

[3] Syngellakis, S. \& Cherukunnath, N., Boundary element analysis of symmetrically laminated plates. Engineering Analysis with Boundary Elements, 28(9), pp. 1005-1016, 2004.

[4] Becker, W., Concentrated forces and moments on laminates with bending extension coupling. Composite Structures, 30, pp. 1-11, 1995.

[5] Hwu, C., Green's function for the composite laminates with bending extension coupling. Composite Structures, 63, pp. 283-292, 2004.

[6] Hwu, C., Boundary integral equations for general laminated plates with coupled stretching-bending deformation. The Royal Society, Proceedings: Mathematical, Physical and Engineering Sciences, 466(2116), 2010.

[7] Courant, R. \& Hilbert, D., Methods of Mathematical Physics vol. II: Partial Differential Equations, Interscience Publishers: New York, 1953.

[8] Hildebrand, F.B., Advanced Calculus for Applications, Prentice-Hall, Inc.: Englewood Cliffs, N.J., 1962.

[9] Cheng, Z.-Q. \& Reddy, J.N., Octet formalism for Kirchhoff anisotropic plates. The Royal Society, Proceedings: Mathematical, Physical and Engineering Sciences, 458(2022), pp. 1499-1517, 2002.

[10] Timoshenko, S.P. \& Woinowsky-Kreiger, S., Theory of Plates and Shells, McGraw-Hill: New York, 1964. 